\title{
Role of uterine artery Doppler indices for predicting intrauterine pathology inconclusive on 2-dimensional transvaginal imaging among infertile women undergoing frozen embryo transfer cycle
}

\section{Lavi Sindhu*}

Department of Reproductive Medicine and IVF, Medicover Fertility, Panchsheel Park, New Delhi, India

Received: 27 October 2021

Accepted: 24 November 2021

\section{*Correspondence:}

Dr. Lavi Sindhu,

E-mail: lavisindhu20@gmail.com

Copyright: (C) the author(s), publisher and licensee Medip Academy. This is an open-access article distributed under the terms of the Creative Commons Attribution Non-Commercial License, which permits unrestricted non-commercial use, distribution, and reproduction in any medium, provided the original work is properly cited.

\begin{abstract}
Background: Objective of current study was to correlate uterine artery Doppler parameters with endometrial findings on hysteroscopy and its importance for the prediction of intrauterine pathology inconclusive on 2-dimensional transvaginal imaging prior to frozen embryo transfer.

Methods: This was a retrospective study conducted at Medicover fertility center, New Delhi, India. The study population comprised of women with infertility who have undergone hysteroscopy before frozen embryo transfer. Total 70 patients were recruited based on inclusion/exclusion criteria. Intrauterine pathology found in $57.2 \%(n=40)$ patients and $42.8 \%(n=30)$ had normal endometrial cavity on hysteroscopy, they were categorized as group 1 and group 2 respectively based on their findings. Both the groups were compared for pre hysteroscopy uterine artery Doppler parameters, resistivity index and pulsatility index. Further clinical pregnancy rate and miscarriage rate were determined in both groups.

Results: Both the groups were comparable for age, body mass index, duration, and type of infertility. Mean RI and PI was significantly higher in group 1 as compared to group $2(0.90 \pm 0.025,2.89 \pm 0.291$ vs. $0.76 \pm 0.043,1.82 \pm 0.27 ; \mathrm{p}$ value $<0.001)$. The best cut-off value of uterine artery RI was $\geq 0.87$ with sensitivity of $90 \%(95 \% \mathrm{CI}=0.7634-0.9721)$, specificity of $100 \%(95 \% \mathrm{CI}=0.8843-1.0000)$ and PI was 2.46 with sensitivity of $100 \%(95 \% \mathrm{CI}=0.9119-1.0000)$, specificity of $96.7 \%$ (95\% CI=0.8278-0.9992).

Conclusions: Uterine artery Doppler is a non-invasive, cost- effective useful add-on tool for routine endometrial evaluation and can be a good predictor for screening intrauterine pathology and valuable in decision making for preIVF hysteroscopy.
\end{abstract}

Keywords: Uterine artery Doppler, Embryo transfer, Endometrial pathology, Hysteroscopy

\section{INTRODUCTION}

Despite new advancements in IVF treatment like improved embryo culture conditions, modified ovarian stimulation protocols, and better cryopreservation techniques, the implantation potential of good quality embryos remains low. ${ }^{1}$ Successful embryo implantation requires synchronous development of good quality embryos along with healthy receptive endometrium and a close dialogue between them. Different methods have been developed to evaluate endometrial receptivity, such as the histological dating of an endometrium after biopsy, the study of endometrial cytokines in uterine flushing, the genomic study of specific genes to assess receptivity in a timed endometrial biopsy and examination of the endometrium through 2D/3D transvaginal scan (TVS). ${ }^{2-4}$ It is a well established fact that intrauterine pathology can affect the implantation of good quality embryos and 
consequent reduction in pregnancy rates of women undergoing IVF. ${ }^{5,6}$ Currently, it is recommended to carefully assess for intrauterine pathology before doing IVF-ET. ${ }^{7-10}$ Transvaginal ultrasonography plays a vital role in fertility treatment and evaluation of the endometrium. Blood flow to the uterine and ovarian artery undergoes important changes corresponding to cyclical hormone changes and can be easily detected using color Doppler. Recently endometrial and uterine blood flow has gained attention to predict the pregnancy outcome after IVF. Uterine blood flow has been found to be an important predictor for successful implantation and pregnancy outcome in many studies. ${ }^{11-13}$ High impedance to uterine blood flow is associated with unexplained infertility, poor endometrial receptivity and, recurrent miscarriages. ${ }^{14-16}$ Optimum uterine blood flow is an important contributor to good endometrial receptivity. Studies have been done to compare conception and nonconception cycles of women for the difference in uterine blood flow. ${ }^{15,17}$ Intrauterine pathology can impair normal blood flow to the uterus and could be detrimental to endometrial receptivity. Keeping this in mind this study was conducted to determine the endometrial status by hysteroscopy and its correlation with uterine artery Doppler findings. Hysteroscopy is considered to be a gold standard for evaluating the endometrial cavity as this is the only method to look into the endometrium closely, observing physiological and pathological patterns, taking a biopsy for histopathology/endometritis, and simultaneously doing treatment in the same setting. ${ }^{18}$ Althogh the reported prevalence of minor intrauterine pathology identified by hysteroscopy is as high as 30 to $45 \%$ even with normal TVS, and significantly higher in patients having previous assisted reproductive techniques failure still the use of routine hysteroscopy before IVF is debatable and there is no consensus on the effectiveness of hysteroscopic surgery in improving pregnancy outcomes. ${ }^{19-21}$ A number of studies had shown increased pregnancy rates after pre-IVF hysteroscopy, whereas some have shown no potential benefits. ${ }^{21-27}$

\section{Aim and objectives}

This study was aimed to assess the role of Doppler analysis of uterine blood flow in predicting intrauterine pathology and its usefulness in deciding the need for hysteroscopy before embryo transfer in selected patients to improve the results.

\section{METHODS}

\section{Study design and duration}

Current study was a retrospective study conducted from June 2020 to April 2021.

\section{Inclusion criteria}

Women undergone hysteroscopy procedure before FET, age $<40$ years, endometrium thickness (ET) $<7 \mathrm{~mm}$, endometrial and uterine artery Doppler records available were included in the study.

\section{Exclusion criteria}

Women with age $\geq 40$ years, adenomyosis, uterine fibroids, and endometriosis were excluded.

\section{Procedure}

Data of all patients undergone hysteroscopy before IVF during the study period at Medicover fertility center, New Delhi was analyzed and screened for inclusion/exclusion criteria. This period was chosen since uniformity in doing uterine artery Doppler before embryo transfer was attained from this period onwards. Out of 82 patients, 70 patients were included in the study based on inclusion/exclusion criteria. Their findings were noted from history and treatment records. Only self -IVF stimulation with antagonist protocol and frozen embryo transfer cycles with good-quality embryos were included. Clinical pregnancy was defined as ultrasonographic visualization of one or more gestational sacs after a positive $\beta$-HCG $(>25 \mathrm{miu} / \mathrm{ml})$ test. Endometrial evaluation and uterine Doppler study procedure details: 2D TVS, using Samsung SonoAce R7, EVN4-9 transvaginal probe $(6.5 \mathrm{MHz})$ was performed by a trained gynecologist and a reproductive medicine specialist. For all patients oral estradiol valerate $2 \mathrm{mg}$ thrice a day was given from day 3 of a cycle for at least 10 days and endometrial evaluation was done. If Endometrial thickness (ET) was found less than $7 \mathrm{~mm}$ then the dose increased maximum up to $12 \mathrm{mg}$. 2D Doppler analysis of bilateral uterine arteries was done at the level of the internal os. Pulsed Doppler activated after identification of the uterine artery, three continuous regular waves of uterine artery blood flow were recorded after adjustment of insonation angle. Measurement of the resistance index (RI) and the pulsatility index (PI) was done by using a simple semiquantitative method for the assessment of uterine Doppler waveform. For each woman, bilateral uterine arteries Doppler spectral parameters were measured. Mean value of bilateral recordings taken.

\section{Hysteroscopy procedure details}

Indications for performing hysteroscopy were previous failed IVF cycle, absent triple layer pattern or if there was any suspicion of endometrial pathology. Hysteroscopy was performed after patient counseling and written informed consent. Informed consent was also taken for data being used for training and research purposes. Hysteroscopy was done postmenstrual, under intravenous sedation or short general anesthesia as required. Bettocchi hysteroscope $2.9 \mathrm{~mm}$ diameter with 30 degrees, forward oblique view was used and $0.9 \%$ normal saline was used as distention medium with pressure between $80-100 \mathrm{~mm}$ $\mathrm{Hg}$ using a pressure adjustable cuff system. The hysteroscopy records were reviewed for a detailed evaluation of the endocervical canal, endometrial cavity, 
anterior, posterior, and lateral walls, and intrauterine findings were noted as a normal cavity, septum, adhesions, a narrow or tubular cavity, or presence of any endometritis. In the same sitting if abnormalities were found then operative hysteroscopy was done. Adhesiolysis was done using hysteroscopy scissors. Fundal and lateral metroplasty was done if narrow or tubular cavity and septal resection were done using scissors or bipolar electrodes. Endometrial biopsy was obtained and sent for histological examination in all cases. The patients who had hysteroscopic evidence of endometritis, received an appropriate antibiotic course. After septal resection and adhesiolysis, patients were given oral estradiol valerate 4-6 mg daily for 4-6 weeks, as required.

\section{Statistical analysis}

Continuous variables were reported as mean \pm 2 SD and compared using unpaired student's t-test to assess differences in the mean value between two groups for normally distributed variables and the Mann-Whitney test was used for not normally distributed variables. Categorical variables were reported as percentage and compared using Chi-square test. All analysis were done using Statistical Package for Social Sciences (SPSS) and the level of significance was set as $\mathrm{p}$ value less than 0.05 . Receiver operating characteristics curve was plotted to get best cut off value for sensitivity and specificity.

\section{RESULTS}

Among 70 women who underwent hysteroscopy, intrauterine pathology was detected in $57.2 \%$ of patients $(n=40)$ and normal findings were present in $42.8 \%$ of patients $(n=30)$, categorized as group 1 and group 2 respectively. In group 1 , intrauterine adhesions were present in $25.7 \%$ of women $(n=18 / 40)$, septum in $10 \%$ $(n=7 / 40)$, endometritis and scarring in $8.57 \%(n=6 / 40)$, a tubular cavity in $4.28 \% \quad(n=3 / 40)$, polypoidal endometrium in $4.28 \%(n=3 / 40)$, and unicornuate uterus in $4.28 \%(n=3 / 40)$ of them as shown in (Table 1$)$.

Table 1: Hysteroscopy findings of study population.

\begin{tabular}{|lll|}
\hline Hysteroscopy findings & N & $\%$ \\
\hline Intrauterine pathology (group 1) & 40 & 57.2 \\
\hline Adhesions & 18 & 25.7 \\
\hline Septum & 7 & 10 \\
\hline Endometritis & 6 & 8.57 \\
\hline Tubular cavity & 3 & 4.28 \\
\hline Polypoidal endometrium & 3 & 4.28 \\
\hline Uniconuate uterus & 3 & 4.28 \\
\hline $\begin{array}{l}\text { Normal cavity } \\
\text { (Group 2) }\end{array}$ & 30 & 42.8 \\
\hline Total & 70 & 100 \\
\hline
\end{tabular}

An operative hysteroscopy procedure was done to correct intrauterine abnormalities accordingly. Both the groups were comparable for baseline characteristics; including age, BMI, infertility duration, and type of infertility. The mean age of the women was $31.6 \pm 3.49$ years in group 1 and $31.7 \pm 4.06$ years in group 2. Mean BMI was $25.10 \pm 4.12$ and $25.23 \pm 4.24$ in group 1 and 2 respectively. The mean duration of infertility was nearly 5 years in both groups ranging from a minimum of 2 years to a maximum of 18 years. The majority of patients were having primary infertility with nearly one-third of secondary infertility cases in both groups. History of past genital tuberculosis present in $20 \%$ (8/40) cases in group 1 and $13.3 \%(4 / 30)$ cases in group 2. Nearly $25 \%$ of patients had a history of previous failed IVF cycles in both groups. History of abortion/D\&C was present in $17.5 \%$ in group 1 and $6.6 \%$ in group 2. Although percentage of genital tuberculosis and history of abortion/D\&C was higher in group 1 but not statistically significant, may be due to less number of cases in both the groups (Table 2). Mean RI, PI and endometrial thickness of both the groups were $0.90 \pm 0.025$, $2.89 \pm 0.291$ and, $8.04 \pm 1.23 \mathrm{~mm}$ respectively in group1 and $0.76 \pm 0.043,1.82 \pm 0.27$, and $8.6 \pm 0.82$ respectively in Group 2 as shown in (Table 3). Significantly high resistance to blood flow present in Group 1 as compared to group $2(p<0.001)$ as seen in (Figure 1-2), suggesting reduced blood supply to the uterus in the presence of intrauterine pathology.

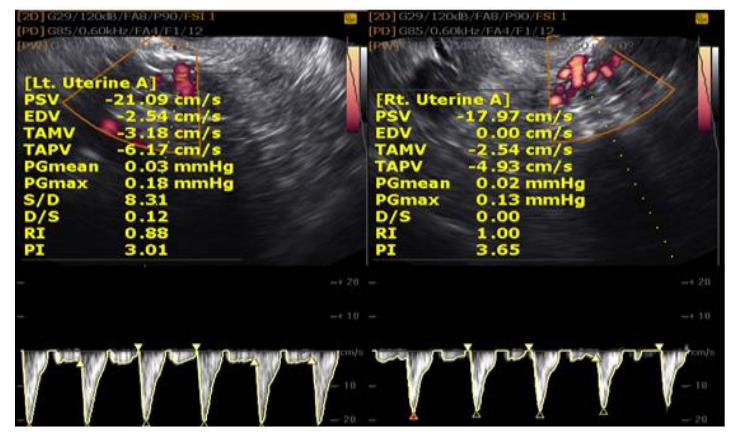

Figure 1: Transvaginal Doppler ultrasound of uterine artery (A left, B right) of women $X$ with intrauterine adhesions showing left and right uterine arteries flow, mean RI and PI 0.94 and 3.33 respectively.

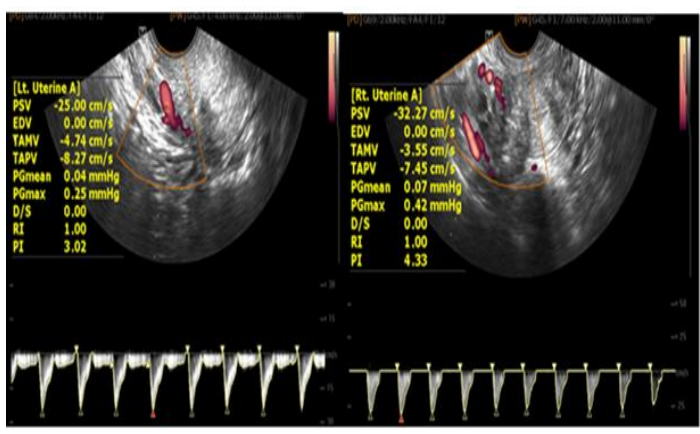

Figure 2: Transvaginal doppler ultrasound of both uterine artery (A left, B right) of women $Y$ with uterine septum showing left and right uterine arteries flow, mean RI and PI 1.0 and 3.67 respectively. 
Good uterine blood flow in group 2 with normal endometrial cavity is depicted in (Figure 3). ROC curve was used to assess the best cut-off value of uterine artery
Doppler indices for the prediction of intrauterine pathology.

Table 2: Comparison of baseline characteristics of in both the groups.

\begin{tabular}{|c|c|c|c|}
\hline Demographic profile & $\begin{array}{l}\text { Group } 1 \\
(\mathrm{~N}=40)\end{array}$ & $\begin{array}{l}\text { Group-2 } \\
(\mathbf{N}=30)\end{array}$ & Significance level \\
\hline Age (years) & $31.6 \pm 3.49$ & $31.7 \pm 4.06$ & 0.440 \\
\hline BMI $\left(\mathrm{Kg} / \mathbf{m}^{2}\right)$ & $25.10 \pm 4.12$ & $25.23 \pm 4.24$ & 0.421 \\
\hline Duration of infertility (years) & $5.5 \pm 2.21$ & $5.2 \pm 3.77$ & 0.492 \\
\hline Primary infertility (\%) & 62.5 & 65 & 0.412 \\
\hline History of tuberculosis (\%) & $08(20)$ & $04(13.3)$ & 0.463 \\
\hline Previous abortion/D\&C (\%) & 0717.5 & $02(6.6)$ & 0.180 \\
\hline Previous 1 or more failed IVF cycle (\%) & $10(17.5)$ & $11(27.5)$ & 0.291 \\
\hline
\end{tabular}

Continuous variables presented as mean \pm SD, and discrete variables as N (\%). Significance level calculated using student's t-test for continuous variables, and Chi-square test for comparison between discrete variables, $\mathrm{p}$ value $\leq 0.05$ is considered as significant difference.

Table 3: Comparison of uterine artery Doppler indices, ET and pregnancy outcome among both the groups.

\begin{tabular}{|llll|}
\hline Endometrial receptivity parameters & $\begin{array}{l}\text { Group-1 } \\
\mathbf{N}=\mathbf{4 0}\end{array}$ & $\begin{array}{l}\text { Group-2 } \\
\mathbf{N = 3 0}\end{array}$ & Significance level \\
\hline RI & $0.90 \pm 0.025$ & $\mathbf{0 . 7 6 \pm . 0 4 3}$ & $<0.001$ \\
\hline PI & $2.89 \pm 0.291$ & $1.82 \pm 0.27$ & $<0.001$ \\
\hline ET & $8.04 \pm 1.23$ & $8.6 \pm 0.82$ & 0.447 \\
\hline Clinical pregnancy rate (\%) & $18(45)$ & $16(53.3)$ & 0.489 \\
\hline Miscarriage (\%) & $2(11.1)$ & $2(12.5)$ & 0.900 \\
\hline
\end{tabular}

Continuous variables presented as mean $\pm \mathrm{SD}$, and discrete variables as N (\%). Significance level calculated using student's t-test for continuous variables, and Chi-square test for comparison between discrete variables, $p$ value $\leq 0.05$ is considered as significant difference.

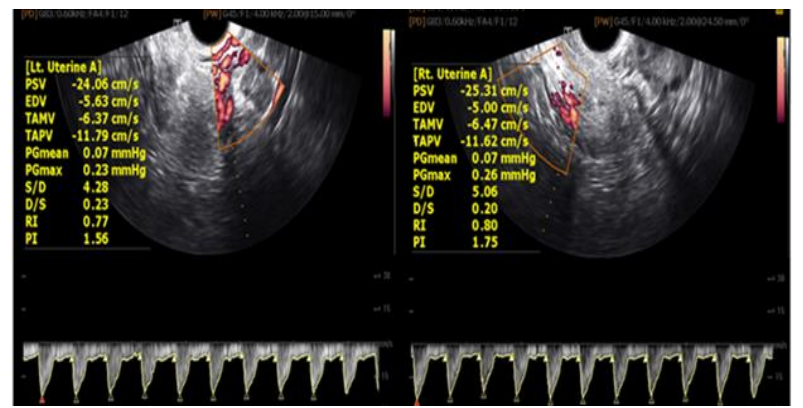

Figure 3: Transvaginal doppler ultrasound of both uterine artery (A left, $B$ right) of women $Z$ with normal uterine cavity showing left and right uterine arteries flow, mean RI and PI 0.78 and 1.65 respectively.

Uterine RI was $\geq 0.87$ with sensitivity of $90 \%(95 \%$ $\mathrm{CI}=0.7634-0.9721), \quad$ specificity of $100 \% \quad(95 \%$ $\mathrm{CI}=0.8843-1.0000$ ), positive predictive value of $100 \%$, and diagnostics accuracy of $94.3 \%$. Area under the curve is 0.9467 with standard error $0.0314(95 \% \mathrm{CI}=0.8356$ 0.9834 ) as shown in (Figure 4). The best cut-off value for uterine PI was 2.46 with sensitivity of $100 \%(95 \%$ $\mathrm{CI}=0.9119-1.0000), \quad$ specificity of $96.7 \% \quad(95 \%$ $\mathrm{CI}=0.8278-0.9992)$, positive predictive value of $97.6 \%$, and diagnostic accuracy of $98.6 \%$. Area under the curve is 0.9988 with standard error $0.0016(95 \% \mathrm{CI}=0.9858$ -
0.9999) as shown in (Figure 5). Among 70 patients, 34 had clinical pregnancy in the study population. In group 1 , a total of 18 patients conceived and 16 patients conceived in group 2 . The clinical pregnancy rate was marginally higher in group 2 as compared to group 1, $53.3 \%(16 / 30)$, and $45 \%(18 / 40)$ respectively, not statistically significant ( $\mathrm{p}$ value $=0.489$ ). The miscarriage rate was also comparable in both groups. Total 4 patients had miscarriage, $2 / 18(11.1 \%)$ patient aborted in group 1 and $2 / 16(12.5 \%)$ in group $2, \mathrm{p}$ value $=0.900$ (Table 3$)$. In group 1, clinical pregnancy data was further analyzed for different intrauterine pathology after corrective surgery or course of antibiotic as per the case. Uterine septum had the highest clinical pregnancy rate of $71 \%$, followed by $66.6 \%$ in polypoidal endometrium, $38.8 \%$ in intrauterine adhesions, $33 \%$ in a tubular cavity, endometritis, and unicornuate uterus each (Table 4).

\section{DISCUSSION}

The uterine endometrium shows a dynamic growth pattern during the menstrual cycle along with hormonal changes, which is a very unique characteristic. The most important function of the uterine endometrium is to provide a receptive bed to support the implantation of an embryo when it reaches the uterus. An adequately estrogen primed endometrium transforms into a receptive one after exposure to progesterone during the luteal phase 
of the menstrual cycle and this period is referred to as the window of implantation.

Table 4: Subgroup comparison of clinical pregnancy rate.

\begin{tabular}{|llll|}
\hline $\begin{array}{l}\text { Endometrial } \\
\text { findings }\end{array}$ & $\begin{array}{l}\text { Number } \\
(\mathbf{N}=70)\end{array}$ & $\begin{array}{l}\text { Clinical } \\
\text { pregnancy } \\
(\mathbf{N}=34)\end{array}$ & $\%$ \\
\hline $\begin{array}{l}\text { Intrauterine } \\
\text { pathology } \\
\text { (group-1) }\end{array}$ & 40 & 18 & 45 \\
\hline Adhesions & 18 & 7 & 38.8 \\
\hline Septum & 7 & 5 & 71 \\
\hline Tubular cavity & 3 & 1 & 33.3 \\
\hline Endometritis & 6 & 2 & 33.3 \\
\hline Polypoidal & 3 & 2 & 66.6 \\
\hline Unicornuate & 3 & 1 & 33.3 \\
\hline $\begin{array}{l}\text { Normal cavity } \\
\text { (group 2) }\end{array}$ & 30 & 16 & 53.3 \\
\hline
\end{tabular}

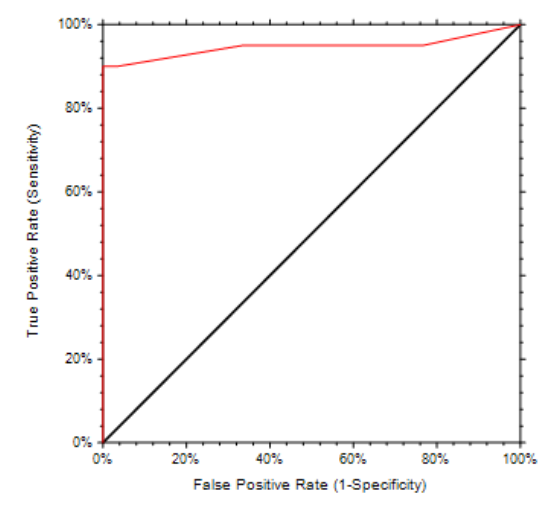

Figure 4: Receiver operating characteristic (ROC) curve for uterine arteries $\mathrm{RI}$ for prediction of intrauterine pathology.

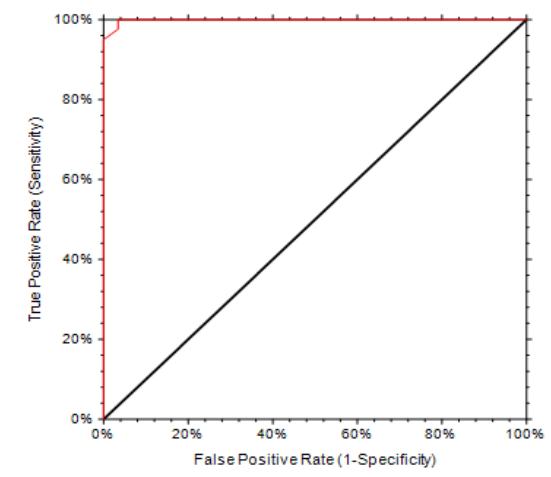

Figure 5: Receiver operating characteristic (ROC) curve for uterine arteries PI for prediction of intrauterine pathology.

Successful implantation depends on close networking between the blastocyst and the receptive endometrium. A good uterine blood flow is usually considered to be an essential requirement for receptive endometrium and normal implantation. ${ }^{28}$ We have chosen hysteroscopy confirmed cases as it is the gold standard method for evaluating uterine cavity and endometrium directly. Even the minor pathology can be detected with great precision, which can be missed with 2D USG. Also, hysteroscopy gives a chance to correct the pathology in the same sitting. In our study confirmed intrauterine pathology was detected in $57.2 \%$ of patients $(n=40)$ and normal findings were present in $42.8 \%$ of patients $(n=30)$ on hysteroscopy. Similar to current study findings, Shawki $\mathrm{HE}$ et al. also found a high proportion (33.3\%) of abnormal uterine findings confirmed by hysteroscopy among patients who had normal HSG and/or TVS. They reported improvement in both implantation and clinical pregnancy rates after hysteroscopy, and recommended it as an important tool for infertility workup before IVF even in patients with normal HSG and/or TVS. ${ }^{8}$ In another recent study unsuspected intrauterine abnormalities were found in $31.6 \%$ of women with unexplained infertility with normal 2D-TVS findings who underwent hysteroscopy before IVF. ${ }^{27}$ Clinical pregnancy rate was higher in women after hysteroscopy as compared to women without hysteroscopy, although the difference was not statistically significant. A prospective study conducted by Chien et al on 633 patients before ET day to assess sub endometrial and endometrial blood flow also confirmed a higher pregnancy rate in patients having good blood flow till endometrium. They concluded that the presence of both sub endometrial and endometrial blood flow is indicative of good endometrial receptivity. Women with advanced age, thin endometrium, and high uterine doppler indices had non detectable blood flow to subendometrium-endometrial region. ${ }^{29}$ During the physiological menstrual cycle, the blood flow of uterine arteries is changed periodically along with hormonal changes. The blood flow impedance is lowest during the luteal phase of the menstrual cycle, resulting in high blood flow to the uterus and endometrium. Hormonal changes and high uterine blood flow at this stage increase the endometrial receptivity and helps in the implantation of embryo. ${ }^{30}$ In the present study, groups were divided based on abnormal and normal hysteroscopic findings and further compared for uterine artery blood flow using 2D-doppler parameters. Both the groups were comparable for age, BMI, infertility duration and type of infertility. Mean uterine artery RI and PI were significantly higher in group 1 with abnormal hysteroscopy findings as compared to group 2 with normal hysteroscopy, suggesting that endometrial pathology like adhesions, endometritis, fibrosis, septum, etc. could be contributing to increased resistance to uterine blood flow among these patients and it can have a negative impact on the endometrial receptivity and implantation potential of the embryo. The best cut off value of RI and PI detected is $\geq 0.87$ and 2.46 respectively with sensitivity of $90 \%$, specificity of $100 \%$ for RI and sensitivity of $100 \%$, specificity of $96.7 \%$ for PI.

To date, there is no study reported on the direct correlation between uterine artery Doppler with 
intrauterine pathology but there are many studies done to compare uterine artery doppler in women with unexplained infertility and fertile controls. They found higher impedance of uterine and spiral arteries in women with unexplained infertility impedance than that of fertile women, and the decrease in the perfusion of the uterus postulated as a cause of unexplained infertility. ${ }^{16,30,31} \mathrm{~A}$ recent comparative study by Zarad et al found significantly higher uterine artery Doppler indices in women with unexplained infertility as compared to fertile women. ${ }^{16}$ The mean value of RI and PI was significantly higher in the infertile group as compared to the fertile group. They predicted cut off values of $\geq 0.67$ and 1.95 for RI and PI respectively for increased resistance to uterine blood flow with sensitivity of 95\%-100\%, specificity of $86.7 \%-96.7 \%$. A study done by Pace et al to compare uterine Doppler indices before and after metroplasty in women with septate uterus. They found a significantly lower value of the mean uterine PI after surgery $(2.07 \pm 0.61$ and $1.49 \pm 0.24$ before and after metroplasty respectively, $\mathrm{p}<0.03$ ). They suggested that metroplasty makes the uterine cavity wider and leads to better uterine perfusion. ${ }^{32}$ Another study done by the same group found significantly lower RI and PI after metroplasty compared doppler indices of uterine arteries before metroplasty. ${ }^{33}$ A possible explanation given to this observation was that septum resection may lead to better vascularization of the endometrium with consequently increased sensitivity to hormones. To the best of our knowledge, this is the first study to show the association between abnormal uterine Doppler and intrauterine pathology. As in this study only infertile patients were taken so comparison with fertile women could not be commented. Because of its retrospective design, this study has certain limitations like Doppler evaluation not done after hysteroscopy, and lesser sample size of study population.

\section{CONCLUSION}

This study concluded that uterine artery Doppler is a noninvasive, cost effective valuable tool in predicting inconclusive endometrial pathology and it can be used in routine practice as an adjunct to routine 2-dimensional TVS for endometrial evaluation. Patients with high uterine RI and PI can benefit by doing hysteroscopy in achieving good implantation and clinical pregnancy rate. As no direct evidence available apart from the current study so we recommend further larger prospective randomized control studies to prove the association and future studies should also look into change in Doppler indices after correction of endometrial pathology and impact on clinical pregnancy and live birth rate.

\section{Funding: No funding sources} Conflict of interest: None declared

Ethical approval: The study was approved by the Institutional Ethics Committee

\section{REFERENCES}

1. Nygren KG, Andersen AN, European IVFmp. Assisted reproductive technology in Europe, 1998. Results generated from European registers by ESHRE. European Society of Human Reproduction and Embryology. Hum Reprod. 2001;16(11):245971.

2. Noyes RW, Hertig AT, Rock J. Dating the endometrial biopsy. Am J Obstet Gynecol. 1975; 122(2):262-3.

3. Ledee-Bataille N, Lapree-Delage G, Taupin JL. Concentration of leukaemia inhibitory factor (LIF) in uterine flushing fluid is highly predictive of embryo implantation. Hum Reprod. 2002;17(1):213-8.

4. Horcajadas JA, Pellicer A, Simon C. Wide genomic analysis of human endometrial receptivity: new times, new opportunities. Hum Reprod Update. 2007;13(1):77-86.

5. Schiano A, Jourdain O, Papaxanthos A. The value of hysteroscopy after repeated implantation failures with in vitro fertilization. Contracept Fertil Sex. 1999;27(2):129-32.

6. Gavino-Gavino F, Guzman-Gonzalez E, ReyesMunoz E. Impact of office hysteroscopy in patients with a history of two or more failed cycles of IVFET and pre-ICSI in assisted an reproduction center. Ginecol Obstet Mex. 2010;78(1):9-14.

7. Fatemi HM, Popovic-Todorovic B. Implantation in assisted reproduction: a look at endometrial receptivity. Reprod Biomed. 2013;27(5):530-8.

8. Shawki HE, Elmorsy M, Eissa MK. Routine office hysteroscopy prior to ICSI and its impact on assisted reproduction program outcome: A randomized controlled trial. Middle East Fertil Soc J. 2012; 17(1):14-21.

9. Dalal RJ. Role of hysteroscopy prior to assisted reproductive techniques in patients with previous IVF Failure. Int J Infertil Fetal Med. 2011;2(1):1922.

10. Shohayeb A, El-Khayat W. Does a single endometrial biopsy regimen (S-EBR) improve ICSI outcome in patients with repeated implantation failure? A randomised controlled trial. Eur J Obstet Gynecol Reprod Biol. 2012;164(2):176-9.

11. Salle B, Bied-Damon V, Benchaib $M$, et al. Preliminary report of an ultrasonography and colour Doppler uterine score to predict uterine receptivity in an in-vitro fertilization programme. Hum Reprod. 1998;13(6):1669-73.

12. Steer CV, Tan SL, Dillon D. Vaginal color Doppler assessment of uterine artery impedance correlates with immunohistochemical markers of endometrial receptivity required for the implantation of an embryo. Fertil Steril. 1995;63(1):101-8.

13. Cacciatore B, Simberg N, Fusaro $\mathrm{P}$, et al. Transvaginal Doppler study of uterine artery blood flow in in vitro fertilization-embryo transfer cycles. Fertil Steril. 1996;66(1):130-4. 
14. Goswamy RK, Steptoe PC. Doppler ultrasound studies of the uterine artery in spontaneous ovarian cycles. Hum Reprod. 1988;3(6):721-6.

15. Steer CV, Campbell S, Tan SL, et al. The use of transvaginal color flow imaging after in vitro fertilization to identify optimum uterine conditions before embryo transfer. Fertil Steril. 1992;57(2):3726.

16. Ali zarad C, Mohamed MH, Shanab WSA. Role of uterine artery Doppler in assessment of unexplained infertility. Egyptian J Radiol Nuclear Med. 2021; 52(1):59.

17. Strohmer H, Herczeg $C$, Plockinger $B$, et al. Prognostic appraisal of success and failure in an in vitro fertilization program by transvaginal Doppler ultrasound at the time of ovulation induction. Ultrasound Obstet Gynecol. 1991;1(4):272-4.

18. Gao M, Sun Y, Xie H. Hysteroscopy prior to repeat embryo transfer may improve pregnancy outcomes for asymptomatic women with repeated implantation failure. J Obstet Gynaecol Res. 2015;41(10):156976.

19. Oliveira FG, Abdelmassih VG, Diamond MP. Uterine cavity findings and hysteroscopic interventions in patients undergoing in vitro fertilization-embryo transfer who repeatedly cannot conceive. Fertil Steril. 2003;80(6):1371-5.

20. Moini A, Kiani K, Ghaffari F. Hysteroscopic findings in patients with a history of two implantation failures following in vitro fertilization. Int J Fertil Steril. 2012;6(1):27-30.

21. Cenksoy P, Ficicioglu C, Yildirim G. Hysteroscopic findings in women with recurrent IVF failures and the effect of correction of hysteroscopic findings on subsequent pregnancy rates. Arch Gynecol Obstet. 2013;287(2):357-60.

22. Elsetohy KA, Askalany AH, Hassan M. Routine office hysteroscopy prior to ICSI vs. ICSI alone in patients with normal transvaginal ultrasound: a randomized controlled trial. Arch Gynecol Obstet. 2015;291(1):193-9.

23. Doldi N, Persico P, Di Sebastiano F. Pathologic findings in hysteroscopy before in vitro fertilizationembryo transfer (IVF-ET). Gynecol Endocrinol. 2005;21(4):235-7.

24. Smit JG, Kasius JC, Eijkemans MJC. Hysteroscopy before in-vitro fertilisation (inSIGHT): a multicentre, randomised controlled trial. Lancet. 2016; 387(10038):2622-9.
25. El-Toukhy T, Campo R, Khalaf Y. Hysteroscopy in recurrent in-vitro fertilisation failure (TROPHY): a multicentre, randomised controlled trial. Lancet. 2016;387(10038):2614-21.

26. Yu HT, Wang CJ, Lee CL. The role of diagnostic hysteroscopy before the first in vitro fertilization/intracytoplasmic sperm injection cycle. Arch Gynecol Obstet. 2012;286(5):1323-8.

27. Singla SGS, Munjal R, Chawla S, Dash BB. Role of pre IVF hysteroscopy in women with unexplained infertility for detecting unsuspected intrauterine lesions and effect on pregnancy outcome. Fertil Sci Res. 2020;7:105-10.

28. Ng EH, Chan CC, Tang OS. Endometrial and subendometrial vascularity is higher in pregnant patients with livebirth following ART than in those who suffer a miscarriage. Hum Reprod. 2007; 22(4):1134-41.

29. Chien LW, Au HK, Chen PL. Assessment of uterine receptivity by the endometrial-subendometrial blood flow distribution pattern in women undergoing in vitro fertilization-embryo transfer. Fertil Steril. 2002;78(2):245-51.

30. Uysal S, Ozbay EP, Ekinci T. Endometrial spiral artery Doppler parameters in unexplained infertility patients: is endometrial perfusion an important factor in the etiopathogenesis? J Turk Ger Gynecol Assoc. 2012;13(3):169-71.

31. Elkholy HANK, Hashad AMN-E, Ibrahim IM. A Study of Endometrial Perfusion in Unexplained Infertility. Egyptian J Hosp Med. 2018;72(6):467380.

32. Pace S, Cerekja A, Stentella P. Improvement of uterine artery Doppler velocimetry indices after metroplasty in arcuate uteri. Eur J Obstet Gynecol Reprod Biol. 2007;131(1):81-4.

33. Pace S, Cerekja A, Dillon KC. Improvement in uterine artery doppler indices via hysteroscopic metroplasty. J Prenat Med. 2013;7(2):25-8.

Cite this article as: Sindhu L. Role of uterine artery Doppler indices for predicting intrauterine pathology inconclusive on 2-dimensional transvaginal imaging among infertile women undergoing frozen embryo transfer cycle. Int J Reprod Contracept Obstet Gynecol 2022;11:80-6. 